\title{
ARTICLE
}

Received 23 Oct 2014 | Accepted 27 Jan 2015 | Published 3 Mar 2015

DOI: $10.1038 /$ ncomms7419

OPEN

\section{Insulating state in tetralayers reveals an even-odd interaction effect in multilayer graphene}

\author{
Anya L. Grushina ${ }^{1}$, Dong-Keun Ki ${ }^{1}$, Mikito Koshino ${ }^{2}$, Aurelien A.L. Nicolet ${ }^{3}$, Clément Faugeras $^{3}$, \\ Edward McCann ${ }^{4}$, Marek Potemski ${ }^{3} \&$ Alberto F. Morpurgo ${ }^{1}$
}

Close to charge neutrality, the electronic properties of graphene and its multilayers are sensitive to electron-electron interactions. In bilayers, for instance, interactions are predicted to open a gap between valence and conduction bands, turning the system into an insulator. In mono and (Bernal-stacked) trilayers, which remain conducting at low temperature, interactions do not have equally drastic consequences. It is expected that interaction effects become weaker for thicker multilayers, whose behaviour should converge to that of graphite. Here we show that this expectation does not correspond to reality by revealing the occurrence of an insulating state close to charge neutrality in Bernal-stacked tetralayer graphene. The phenomenology-incompatible with the behaviour expected from the single-particle band structure-resembles that observed in bilayers, but the insulating state in tetralayers is visible at higher temperature. We explain our findings, and the systematic even-odd effect of interactions in Bernal-stacked layers of different thickness that emerges from experiments, in terms of a generalization of the interaction-driven, symmetry-broken states proposed for bilayers.

\footnotetext{
${ }^{1}$ Department of Quantum Matter Physics (DQMP) and Group of Applied Physics (GAP), University of Geneva, 24 Quai Ernest-Ansermet, CH1211 Genéve 4 Switzerland. ${ }^{2}$ Department of Physics, Tohoku University, Sendai 980-8578, Japan. ${ }^{3}$ Laboratoire National des Champs Magnétiques Intenses, CNRS-UJFUPS-INSA, Grenoble 38042, France. ${ }^{4}$ Department of Physics, Lancaster University, Lancaster, LA1 4YB, UK. Correspondence and requests for materials should be addressed to A.F.M. (email: Alberto.Morpurgo@unige.ch).
} 
C lose to charge neutrality and at zero magnetic field, $\mathbf{B}=0$, electron-electron interactions in graphene and its multilayers have a strong effect in a very narrow range of electron density ${ }^{1}$. In monolayer graphene, Coulomb interactions renormalize the Fermi velocity of the Dirac fermions, which-in agreement with theoretical predictions ${ }^{1}$-increases as the Fermi level approaches the charge neutrality point $(\mathrm{CNP})^{2}$. In Bernal bilayers, interactions are considered to be responsible for the opening of a gap ${ }^{1,3-7}$, and they turn the system into an insulator at a low temperature ${ }^{8-11}$. Thicker Bernal-stacked multilayers have received less attention, and it is only known that Bernal trilayers remain conducting at low temperature (rhombohedral trilayerson the contrary-have again been found to open a large gap due to interactions) ${ }^{12}$. In all cases-that is, for mono and bilayersthe effects of interactions are visible experimentally only at charge densities below $2-3 \times 10^{10} \mathrm{~cm}^{-2}$, and their observation requires the study of suspended devices of the highest quality ${ }^{13,14}$, since otherwise the magnitude of charge inhomogeneity is too large.

Here we realize very high-quality multiterminal suspended devices based on Bernal-stacked tetralayer graphene (4LG), in which charge inhomogeneity can be reduced to the level of the best mono and bilayer structures realized in the past. Thanks to their very high quality enabled by employing a recently developed fabrication process (see ref. 15 and Methods for details), these devices allow us to reveal experimentally the occurrence of a very pronounced and robust insulating state at the CNP in 4LG, clearly visible already at relatively high temperature (at $T=4.2 \mathrm{~K}$ the resistance at the CNP is several hundreds of kiloOhms, 50 times larger than in analogous bilayer devices). We find that the size of the energy gap in 4LG coincides with the values reported previously for bilayers, strongly suggesting a common origin for the insulating state in the two system. We discuss the phenomenon theoretically, and suggest that a symmetry-broken state in which a staggered potential is present in $4 \mathrm{LG}-$ corresponding to the simplest generalization of the symmetrybroken states that are considered to explain the behaviour of bilayers-naturally explains our observations, as well as the coincidence between the values of energy gap observed in 4LG and bilayers. These same theoretical considerations point to an even-odd interaction effect in Bernal-stacked graphene multilayers at the CNP (odd multilayers remain conducting at low temperature, whereas even multilayers become insulating) that is consistent with all experimental observations so far, and has implication for the crossover from graphene to graphite.

\section{Results}

Highly resistive state in charge neutral Bernal-stacked 4LG. Before discussing the detailed characterization of the thickness and the stacking of the layers in our devices, we present the experimental indications of the high device quality and of the unusual highly resistive state at the CNP, which can be appreciated already in the most basic transport measurements. Figure 1a shows the gate voltage $\left(V_{\mathrm{G}}\right)$ dependence of the twoterminal resistance $\left(R_{2-4}\right)$ of two different devices measured at $4.2 \mathrm{~K}$. In both cases, a very high and narrow peak is observed around the $\mathrm{CNP}, \sim 50$ times higher than that observed in suspended monolayer and Bernal-stacked bi/trilayer graphene at the same temperature $e^{2,8-16}(\sim 0.3 \mathrm{M} \Omega$ as compared with $4-8 \mathrm{k} \Omega$; see Supplementary Fig. 1; the two devices exhibit identical behaviour and in the rest of the manuscript we show the data from one of them). The peak width is extracted from the log-log plot of the conductance $G_{2-4}=1 / R_{2-4}$ versus carrier density $n$, as illustrated in Fig. $1 \mathrm{~b}$ ( $n$ is calculated from the applied $V_{\mathrm{G}}$, with the gate capacitance obtained from the analysis of the quantum Hall effect; see discussion of Fig. 2d). The range in which the
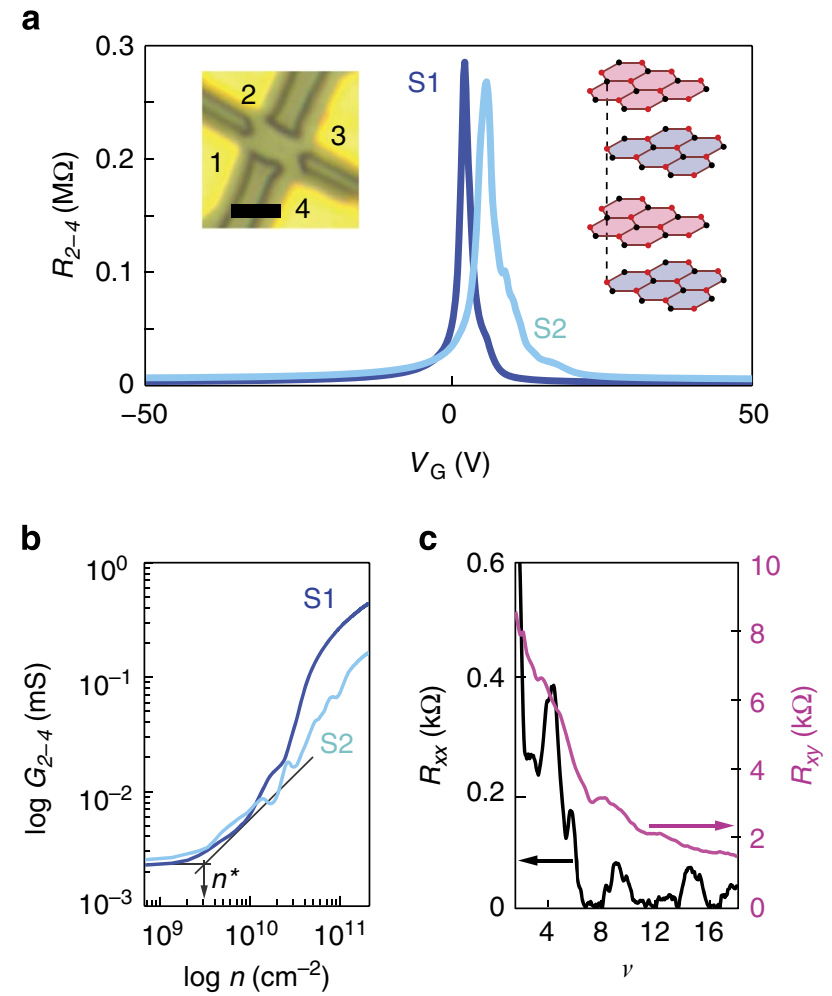

Figure 1 | Very high resistance at charge neutrality in high-quality suspended tetralayer graphene devices. (a) $V_{G}$ dependence of the twoterminal resistance $R_{2-4}$ measured in two devices (S1 and S2) at $4.2 \mathrm{~K}$, showing a peak resistance $\sim 0.3 \mathrm{M} \Omega$ at the CNP, much larger than the value normally observed in mono or BLG at the same temperature $\left(R_{\alpha-\beta}\right.$ represents the two-terminal resistance measured between contacts $\alpha$ and $\beta$, with the indexes labelling the contacts shown in the left inset). Left inset: optical microscope image of a device covered by a PMMA mask before etching (see Methods; scale bar, $2 \mu \mathrm{m}$ long). Right inset: crystal structure of Bernal-stacked 4LG. (b) Conductance $G_{2-4}=1 / R_{2-4}$ as a function of $n$ in a double-logarithmic scale, showing a very low level of charge inhomogeneity, $n^{*}=2-3 \times 10^{9} \mathrm{~cm}^{-2}$ (pointed to by the arrow). The density is given by $n=\alpha\left(V_{\mathrm{G}}-V_{\mathrm{CNP}}\right)$, with $\alpha=4.66 \times 10^{9} \mathrm{~cm}^{-2} \mathrm{~V}^{-1}$ determined from the analysis of QHE (see Fig. 2d). (c), Fully developed QHE measured at $\mathbf{B}=0.45 \mathrm{~T}$, with vanishing longitudinal resistance $R_{x x}=R_{1-4,2-3}$ (black curve) and quantized transverse resistance $R_{x y}=R_{1-3,4-2}=1 / v \times h / e^{2}$ (magenta curve) at $v=8,12$ and 16 (data taken at $250 \mathrm{mK} ; R_{\alpha-\beta, \gamma-\delta}$ corresponds to the ratio of the voltage difference measured between contacts $\gamma$ and $\delta$, and the current flowing from contact $\alpha$ to $\beta$ ).

conductance stays constant upon increasing $n-$ a measure of the carrier density fluctuations ${ }^{13,14}$-extends only up to $n^{*} \sim 2$ $3 \times 10^{9} \mathrm{~cm}^{-2}$, comparable to the best values reported in the literature for suspended graphene of any thickness ${ }^{2,8-16}$. Finally, the multiterminal configuration allows magnetotransport to be measured in both longitudinal and transverse configurations ${ }^{15,16}$. Already at moderately low $\mathbf{B}$, a fully developed integer quantum Hall effect (IQHE) is observed (see Fig. 1c), with integer conductance plateaus (in units of $e^{2} / h$ ) in the transverse resistance $\left(R_{x y}\right)$ and concomitant vanishing of the longitudinal resistance $\left(R_{x x}\right)$.

The thickness and stacking order of our samples are determined by magneto-Raman spectroscopy. The 2D Raman peak, occurring around $2,710 \mathrm{~cm}^{-1}$ at $\mathbf{B}=0$ when measured with an excitation at $\lambda=514 \mathrm{~nm}$, exhibits the contribution of two components (see inset of Fig. 2a), as expected for 4LG with Bernal stacking ${ }^{17-19}$. This observation, however, is not sufficient to 
B ( $\mathrm{T})$
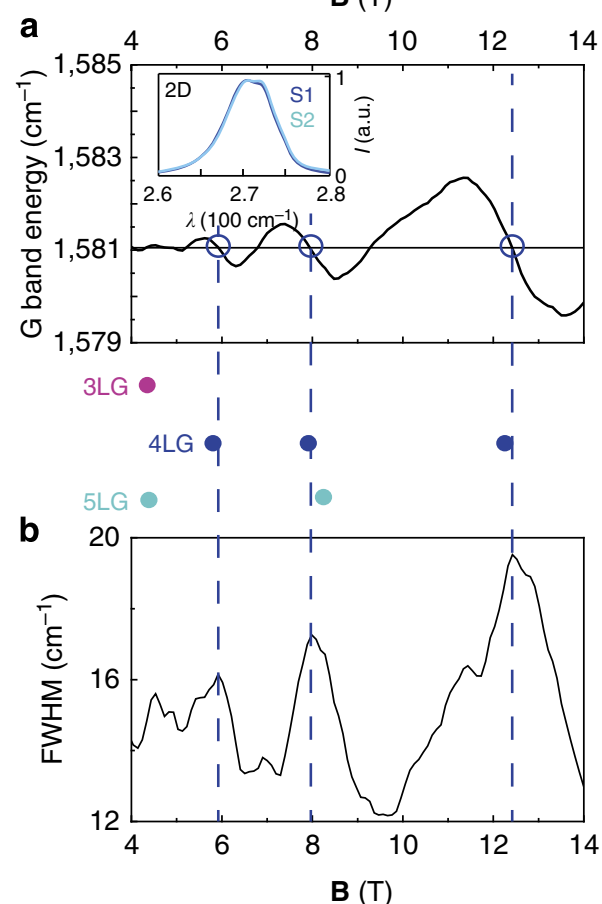
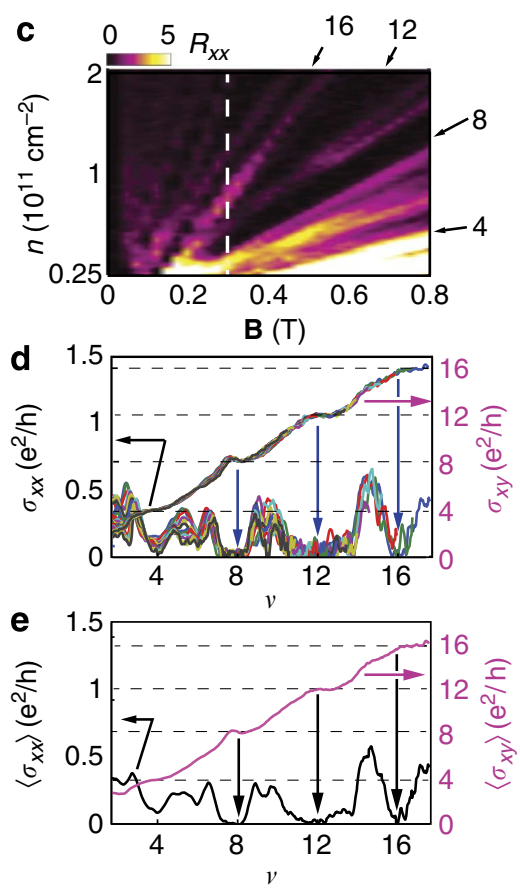

Figure 2 | Magneto-Raman spectroscopy and four-probe quantum Hall transport in suspended tetralayer graphene. (a) Position of the Raman G-band peak as a function of $\mathbf{B}$ measured at room temperature on one of our suspended devices, exhibiting characteristic magneto-Raman oscillations (the inset shows the 2D Raman peak at zero B). The empty circles denote the position at which the anti-crossings occur, which coincide at values of $\mathbf{B}$ for which the broadening of the Raman G line (measured by the full width at half maximum-FWHM-shown in (b)) peaks. The magenta, blue and cyan circles between panels $\mathbf{a}$ and $\mathbf{b}$ correspond to the values of $\mathbf{B}$ at which the anti-crossings are expected for tri-, tetra- and pentalayer Bernal-stacked grapheme, respectively (see the main text for details): this comparison unambiguously identifies our layer as Bernal-stacked $4 \mathrm{LG}$. (c) $R_{x x}$ plotted as a function of $\mathbf{B}$ and $n$. Clear Shubnikov-de Haas oscillations are visible starting from $\mathbf{B}=0.1 \mathrm{~T}$ and fully developed quantum Hall states from $\mathbf{B}=0.3 \mathrm{~T}$. The arrows indicate the local $R_{x x}$ minima (black strips) at $v=4,8,12$ and 16 (the $v=8$ state is the first to develop fully at $\mathbf{B} \sim 0.3 T$; white dashed line). At low $n$, below $2.5 \times 10^{10} \mathrm{~cm}^{-2}$, the device becomes highly insulating (see Fig. 3), preventing multiterminal measurements. (d) Traces of the longitudinal and transverse conductivities, $\sigma_{x x}=\rho_{x x} /\left(\rho_{x x}^{2}+\rho_{x y}^{2}\right)$ and $\sigma_{x y}=\rho_{x y} /\left(\rho_{x x}^{2}+\rho_{x y}^{2}\right)$, as a function of $v$, measured for $\mathbf{B}$ between $0.475 \mathrm{~T}$ and $0.8 \mathrm{~T}\left(\rho_{x x}=R_{x x} \times W / L\right.$ and $\rho_{x y}=R_{x y}$ with the device aspect ratio $W / L=0.87)$. The filling factor $v=n h / e \mathbf{B}$ is calculated from $\mathbf{B}$ and $n=\alpha\left(V_{G}-V_{C N P}\right)$ with $\alpha=4.66 \times 10^{9} \mathrm{~cm}-2 \mathrm{~V}-1$ determined by imposing scaling of all the measured curves in this magnetic field-range. The good scaling enables averaging of the magnetoconductivity curves to suppress noise, as shown in $\mathbf{e}$.

exclude tri- and pentalayer graphene unambiguously. To gain conclusive evidence, Raman spectra were collected in the regime of Landau quantization, by applying a finite $\mathbf{B}$ to split the singleparticle energy spectrum into discrete Landau levels (LLs). In this regime, the G Raman peak exhibits characteristic anti-crossings upon increasing $\mathbf{B}$-an oscillatory shift of the peak position with a concomitant broadening (see Fig. 2a,b)-when the energy of the $E_{2 g}$ phonon matches that of specific inter-LL transitions (see ref. 20 for details). In few layer graphene, whose electronic spectrum consists of several bands ${ }^{21-27}$, the effect of coupling is enhanced in the presence of nearly degenerate transitions (that is, when inter-LL transitions from different bands occur at nearly the same energy) $)^{28}$, and become measurable even at room temperature (for non-degenerate transitions, the effect of coupling is too weak to be detected). In the simplest approximation-that we will refer to as the effective bilayer model-the single-particle band structure of $4 \mathrm{LG}$ consists of two independent bilayer-like bands with different effective masses $^{21-27}$, and predicts pairs of nearly degenerate inter-LL transitions to match the phonon energy at $\mathbf{B} \sim 5.8,7.8$ and $12 \mathrm{~T}$ as marked by the blue circles between Fig. $2 a-b^{28}$. As indicated by the blue dashed lines in Fig. 2a-b, the observed features are in virtually perfect agreement with these predictions, whereas they do not match the expectations for tri- and pentalayer graphene (respectively, magenta and cyan circles between Fig. 2a,b) nor those for non-Bernal-stacked multilayers (note, in this regard, that since our devices are realized by exfoliating natural graphite, the probability to find two-out-of-two identically non-Bernalstacked 4LG is entirely negligible). Magneto-Raman spectroscopy, therefore, unambiguously identifies our devices as made of Bernal-stacked 4LG. In addition, by showing the persistence of Landau quantization at room temperature, these observation further provide another clear indication of the high quality of our devices.

To characterize the device quality in more detail, and to gain additional understanding of the low-energy electronic properties, we have investigated transport in the quantum Hall regime by taking advantage of the multiterminal geometry that enables both $R_{x x}$ and $R_{x y}$ to be measured ${ }^{15,16}$. Figure $2 c$ shows a colour plot of $R_{x x}\left(V_{\mathrm{G}}, \mathbf{B}\right)$, exhibiting clear features originating from the QHE, with Shubnikov-de Haas oscillations becoming visible from $\mathbf{B} \sim 0.1 \mathrm{~T}$. Through the condition for their visibility $(\mu \mathbf{B} \gg 1$; refs 13,14$)$, we estimate the carrier mobility $\mu$ to be larger than $100,000 \mathrm{~cm}^{2} \mathrm{~V}^{-1} \mathrm{~s}^{-1}$. The oscillations evolve into fully developed IQHE states-with plateaus in $R_{x y}$ and vanishing $R_{x x}$-starting from $\mathbf{B} \sim 0.3 \mathrm{~T}$ at filling factor $v \equiv n h / e \mathbf{B}=8$ (see the white dashed line in Fig. 2c), and subsequently at $v=12$ and 16 (starting from B $\sim 0.4-0.5 \mathrm{~T})$. These states are clearly apparent in Fig. 2d, which shows the longitudinal $\left(\sigma_{x x}\right)$ and transverse $\left(\sigma_{x y}\right)$ conductivity measured as a function of $V_{\mathrm{G}}$ for $\mathbf{B}$ in the range $0.475-0.8 \mathrm{~T}$, and 
plotted as a function of $v$, with the gate capacitance relating $V_{\mathrm{G}}$ and $n$ determined by enforcing the scaling of all curves (note that the good scaling allows us to reduce noise by averaging ${ }^{16}$ as shown in Fig. 2e; it also indicates the absence of significant inhomogeneity in the carrier density close to the edges of the $4 \mathrm{LG}$, as it may be expected from basic electrostatic considerations ${ }^{29}$ ). These fully developed states at low $\mathbf{B}$ follow the sequence expected from the simplest description of the electronic structure of 4LG in terms of two decoupled bilayer-like bands, for which a 16-fold degenerate LL at zero energy needs to be completely filled before electrons occupy (fourfold degenerate) LLs at higher energy ${ }^{25}$. The appearance of the $v=4$ state, which starts from $\mathbf{B}=0.8 \mathrm{~T}$, indicates that, at higher fields, the 16-fold degeneracy of the zero energy LLs starts to break. Possible mechanisms responsible for breaking this degeneracy are the effect of next-nearest-layer hopping ${ }^{30}$ (that is not considered in the effective bilayer model) or electron-electron interactions ${ }^{31}$, for which an analysis of the behaviour at larger $\mathbf{B}$ provides additional evidence (see discussions below). Irrespective of these details, the observation of the 16-fold degenerate zero-energy LL provides useful information about the low-energy electronic properties of suspended $4 \mathrm{LG}$.

Insulating state and electron-electron interaction in 4LG. With the device structure and quality characterized well, we look at the high resistance peak found close to the CNP (Fig. 1a). At base temperature $(T=250 \mathrm{mK}$; see Methods), the conductance $G$ becomes unmeasurably small (the resistance is $>100 \mathrm{M} \Omega$ ), as shown in Fig. 3a. The different curves in the figure correspond to two-terminal measurements done using different pairs of contacts (as indicated in the legend; for these high resistance values, fourterminal measurements cannot be done reliably), and show that the strong suppression always occurs in a same range of carrier density $|n|<2-3 \times 10^{10} \mathrm{~cm}^{-2}$, irrespective of the measurement configuration. This finding is an indication of the very high device homogeneity, which can be checked directly when working with multiterminal devices ${ }^{15,16}$. Figure $3 b$ shows the temperature dependence of $G\left(V_{\mathrm{G}}\right)$ measured in one of the two-terminal configurations (other configurations give identical results), showing a pronounced insulating behaviour upon lowering $T$. Above $1 \mathrm{~K}$, the minimum conductance is thermally activated $G_{\min }=\exp \left(-E_{\mathrm{A}} / 2 k_{\mathrm{B}} T\right)$ with an activation energy $E_{\mathrm{A}} \sim 15 \mathrm{~K}$ (see Fig. 3c), indicating the presence of an energy gap at the CNP. The gap closes very rapidly when the device is gate-biased away from the CNP, as it can be seen in the measurement of the differential conductance $(\mathrm{d} I / \mathrm{d} V)$ as a function of different $V_{\mathrm{G}}$ and applied bias voltage $V_{\mathrm{B}}$ (Fig. 3d). Figure $3 \mathrm{e}$ further shows that at low bias, the $\mathrm{d} I / \mathrm{d} V$ vanishes, within the accuracy of the measurements, for all bias voltages $V_{\mathrm{B}}$ below a threshold $(\sim 1.5 \mathrm{mV}$, corresponding approximately to the activation energy extracted from the $T$ dependent measurements), above which it increases sharply. a

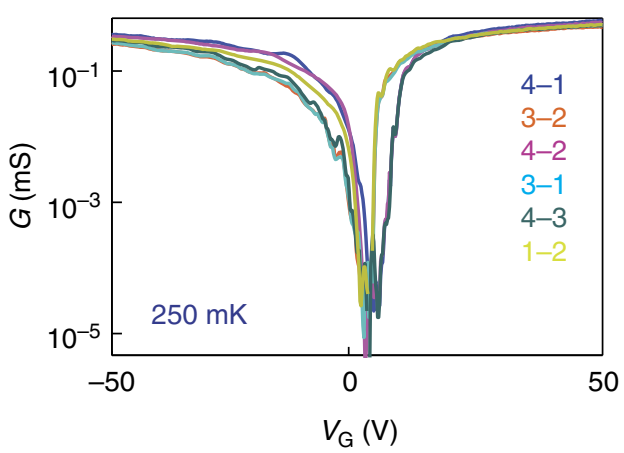

b

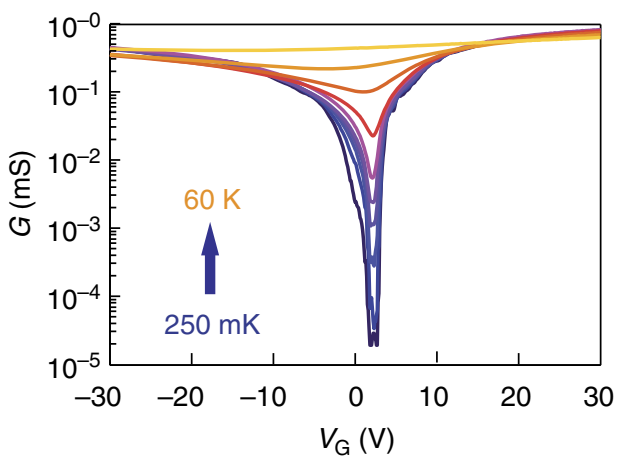

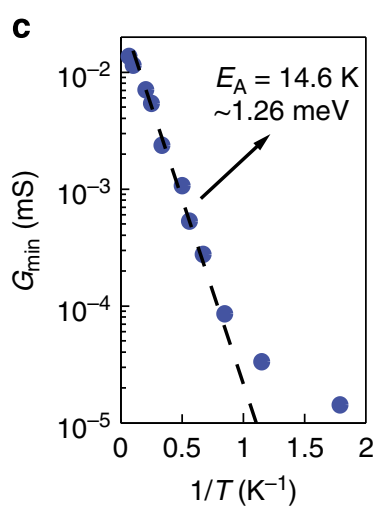
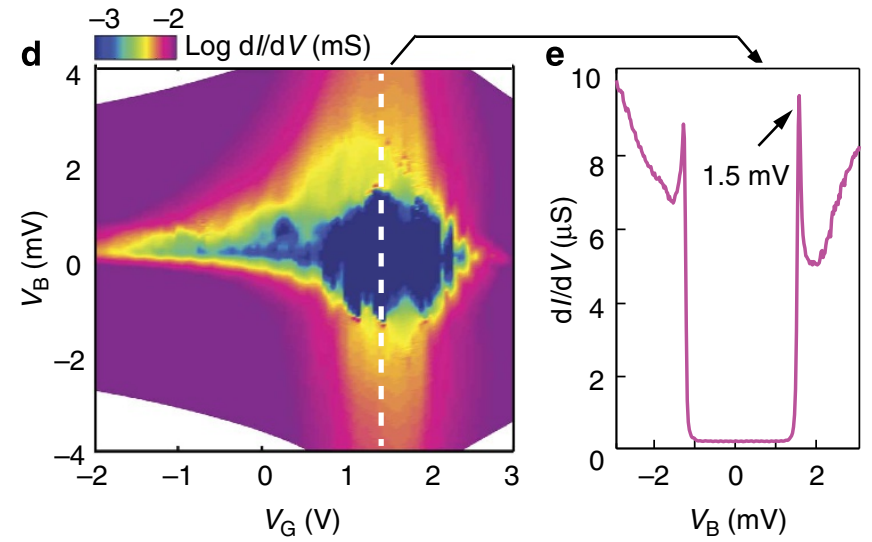

Figure 3 | Insulating state at charge neutrality and $\mathbf{B}=\mathbf{0 T}$ in tetralayer graphene. (a) $\log (G)$ versus $V_{G}$, measured in several different two-terminal configurations, as indicated in the legend (see inset in Fig. 1a for the contact labelling). The same behaviour-a pronounced suppression of $G$ in a very narrow range of gate voltages $\left|V_{G}-V_{C N P}\right|<5 V$, corresponding to $|n|<2 \times 10^{10} \mathrm{~cm}^{-2}$-is observed irrespective of the contacts used, which is indicative of the high device homogeneity. (b) $T$-dependence of $\log (G)$-versus $V_{G}$ measured in a two-terminal configuration. Upon increasing $T$, the minimum conductance $G_{\min }$ at the CNP increases by $>4$ orders of magnitude, exhibiting a thermally activated behaviour for $T>1 \mathrm{~K}$, as shown in panel $\mathbf{c}\left(G_{\min }=\exp (-\right.$ $E_{\mathrm{A}} \sqrt{2} \mathrm{k}_{\mathrm{B}} T$ ) with $E_{\mathrm{A}} \sim 14.6 \mathrm{~K}$ ). (d) Colour plot of the differential conductance $G=\mathrm{d} l / \mathrm{d} V$ (in log scale) as a function of source-drain $\left(V_{B}\right)$ and gate voltage $\left(V_{\mathrm{G}}\right)$, measured at $250 \mathrm{mK}$. The insulating state corresponds to the dark blue region close to the CNP and $V_{B}=0 \mathrm{~V}$. (e) Line-cut, $\mathrm{d} / / \mathrm{d} V\left(V_{B}\right)$, of the colour plot shown in $\mathbf{d}$, taken at $V_{G}=1.6 \mathrm{~V}$ (corresponding to the white dashed line in panel $\mathbf{d}$ ). Below $V_{B} \sim 1.5 \mathrm{mV}$ (indicated by the arrow), the conductance vanishes within the accuracy of the measurement. 
In the case of bilayer graphene (BLG), a qualitatively similar behaviour has been observed ${ }^{8,9}$ (see also Supplementary Note 1) and interpreted in spectroscopic terms, with the applied bias taken to be a direct measure of the energy of the injected electrons $^{8,9}$. It cannot be excluded, however, that the dominant effect of the applied bias is the electrostatic accumulation of charges on the suspended 4LG flake. Indeed, since the gap closes very rapidly upon increasing the charge density away from the CNP, the accumulation of even a very small amount of charges can result in a drastic suppression of the gap, leading to a large and sharp conductance increase.

The occurrence of an insulating state at the CNP is unexpected. It cannot be accounted for in terms of the gapless single-particle band structure of $4 \mathrm{LG}^{21-27}$, and its explanation calls for the effects of electron-electron interactions (a scenario based on the presence of macroscopic defects such as AB-BA stacking faultswhich has been proposed for bilayers ${ }^{32,33}$ to explain why some of high-quality suspended devices remain conducting at low $T$ (ref. 34) - is incompatible with our data, and with different observations reported in the literature; Supplementary Note 2). Indeed, the appearance of fully developed QHE at $\mathbf{B}=2.3 \mathrm{~T}$ with plateaus in $\sigma_{x y}$ at 1,2 and $3 e^{2} / h$ and vanishing $\sigma_{x x}$ (Fig. $4 \mathrm{a}$ ) demonstrates that the interactions are strong enough to completely lift the degeneracy of the zero-energy LL already at low B. Plotting $\mathrm{d} G / \mathrm{d} \mathbf{B}$ as a function of $\mathbf{B}$ and $n$ shows that these symmetry-broken states start developing from $\mathbf{B} \sim 1 \mathrm{~T}$ (Fig. $4 \mathrm{~b}$ ). a

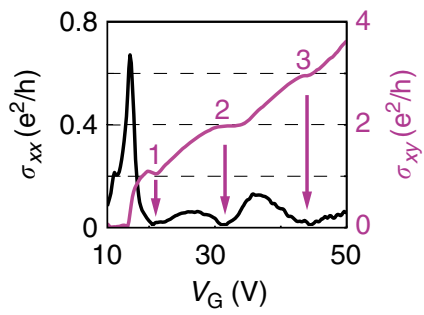

C

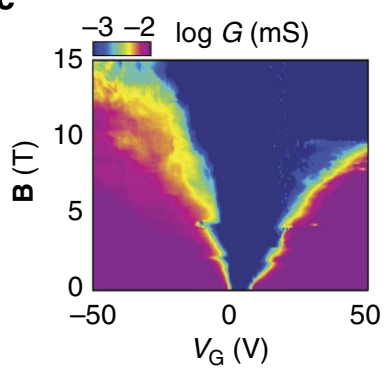

b

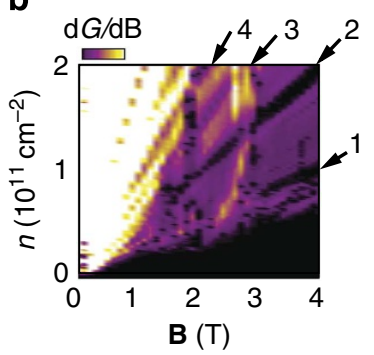

d

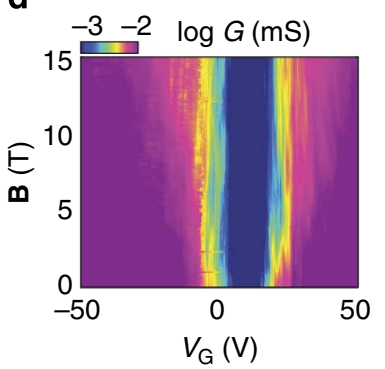

Figure 4 | Strong electron-electron interactions at finite magnetic field. (a) $V_{\mathrm{G}}$ dependence of the longitudinal ( $\sigma_{x x}$ i black curve) and the transverse ( $\sigma_{x y} ;$ magenta curve) conductivity at $\mathbf{B}=2.3 \mathrm{~T}$, showing fully developed broken symmetry states at $v=1,2$ and 3 with zeros in $\sigma_{x x}$ and plateaus in $\sigma_{x y}$. (b) Fan diagram of the derivative of the conductance $d G / d \mathbf{B}(\mathbf{B}, n)$, illustrating the evolution of the quantum Hall states at $v=1,2,3$ and 4 (black strips indicated by arrows) as a function of $\mathbf{B}$ and $n$ : these states survive down to $\sim 1 \mathrm{~T}$ (the vertical features visible at $\mathbf{B}$ around 1.8 and $2.8 \mathrm{~T}$ are likely due to crossing in energy between the different symmetry broken LLs). (c) Upon the application of perpendicular magnetic field, the insulating state (dark blue region close to $\mathrm{CNP}$ at $\mathbf{B}=0 \mathrm{OT}$ ) extends through a much broader range of carrier density, similarly to what happens in mono and BLG. (d) If the magnetic field is applied in the plane, no change is seen in the range of $\mathbf{B}$ accessible in our laboratory (15T). All measurements shown in this figure were taken at $T=250 \mathrm{mK}$.
This value corresponds to the magnetic field at which the $v=4$ state becomes visible, suggesting that the appearance of this state is also due to interactions. Note that, in the fractional quantum Hall effect (likely to be within experimental reach at higher $\mathbf{B}$ with devices of the quality shown here), the 16-fold degeneracy of the zero-energy LL is expected to lead to new unexplored regimes, occurring when states belonging to different LLs are mixed by interactions ${ }^{35-37}$. The first manifestations of these new regimesthe appearance of an even-denominator fractional state at $v=-1 / 2$ (ref. 16) and of electron-hole asymmetry ${ }^{38,39}$-have just been reported in BLG: with its larger degeneracy, 4LG should lead to an even richer spectrum of new phenomena. Finally, additional evidence for the relevance of the interactions in $4 \mathrm{LG}$ is provided by the enhancement of the insulating state around charge neutrality, which at higher magnetic field extends throughout a larger density range (Fig. 4c). This behaviour is identical to that observed in mono and BLG, where it has been shown to originate from a canted anti-ferromagnetic state due to interactions ${ }^{40-42}$ (a similar explanation may be valid for $4 \mathrm{LG}$. Indeed, if we consider the magnitude of the gap $\sim 1.5 \mathrm{meV}$, the data in Fig. 4d, which show that the insulating state is not affected by a parallel magnetic field $\mathbf{B}$ up to $15 \mathrm{~T}$, indicates that the electron spin is not a good quantum number in the insulating state). The finding that the enhancement starts already at low $\mathbf{B}$ (Fig. 4c) and that the insulating state at finite field evolves continuously into the $\mathbf{B}=0$ insulating state upon reducing $\mathbf{B}$, suggests a related origin of both the states.

Gap origin and even-odd effect in graphene multilayers. A comprehensive analysis of the effect of electron-electron interactions in Bernal-stacked 4LG is complex and goes beyond the scope of this paper. However, our observation that the magnetoRaman measurements and the IQHE at low $\mathbf{B}$ are in surprisingly good agreement with the behaviour expected from the effective bilayer model suggests that treating $4 \mathrm{LG}$ by analogy with the case of graphene bilayers is a good starting point. At a mean-field level $\mathrm{e}^{3,5,7,8}$, the low-energy properties of the symmetry-broken states in BLG are commonly described within a two-band model, in terms of a $2 \times 2$ matrix Hamiltonian of the type

$$
H^{B L G}=-\frac{\hbar^{2}}{2 m *}\left(\left(k_{x}^{2}-k_{y}^{2}\right) \sigma_{x} \pm 2 k_{x} k_{y} \sigma_{y}\right)-\Delta \sigma_{z}
$$

for each valley and spin (the ' \pm ' sign changes going from the $K$ to the $\mathrm{K}^{\prime}$ valley). The first term is the kinetic energy of the electrons ( $\mathrm{m}^{*}$ is the effective mass) and the second one represents the order parameter of the broken symmetry state $\left(\sigma_{x, y, z}\right.$ are Pauli matrices acting on the layer degree of freedom). Different ground states emerge, depending on how the sign of the order parameter $\Delta$ changes upon switching valley or $\operatorname{spin}^{7,8}$, but, in all cases, the 'bulk' of BLG is gapped (see Fig. 5a) as the term $\Delta \sigma_{z}$ in the Hamiltonian has an opposite sign in opposite layers for any fixed spin and valley configuration. The analogy with BLG suggests that this mean-field treatment can be extended to the case of 4LG in a natural way, by generalizing the interlayer potential asymmetry in BLG as a staggered potential $V_{i}=(-1)^{i+1} \Delta(i=1, \ldots, 4$ is the layer index) which changes sign between the neighbouring layers. More specifically, the Hamiltonian of $4 \mathrm{LG}$ is decomposed into blocks describing two effective graphene bilayers using a unitary transformation, and the staggered potential then works as the $\Delta \sigma_{z}$ term for each bilayer (see Supplementary Note 3). It is straightforward to show by direct diagonalization of the 4LG Hamiltonian that, within the effective bilayer model, the staggered potential $\Delta$ opens a gap at the CNP in $4 \mathrm{LG}$ (see Fig. 5a; again, in analogy to the BLG, ground states with different properties shown in Supplementary Fig. 2 originate from the way in which $\Delta$ changes upon switching valley or spin). While the 
a

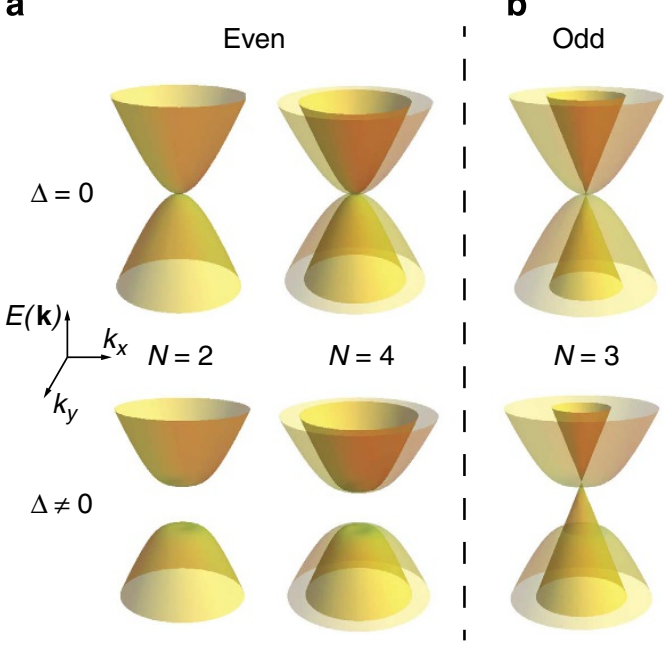

understood why the simple approximation used in this study to describe the Bernal multilayers (which neglects, for instance, next-layer hopping processes, responsible for the $\gamma_{2}$ and $\gamma_{5}$ parameters in the tight-binding description of graphite) correctly captures the basic aspects of the electron-electron interactions. The robustness of the even-odd effect may be rooted in the symmetry of these systems, which is different in the two cases: even multilayers are inversion symmetric, whereas odd ones possess reflection symmetry ${ }^{27}$ (for thickness larger than the monolayer). It therefore seems plausible that the appearance of a staggered potential-which breaks inversion and preserves reflection symmetry-can account for a drastic modification of the behaviour of even multilayers, and not of that of odd ones.

\section{Discussion}

The experimental observation of the even-odd effect just discussed is particularly interesting because it shows how the interactions induce robust, systematic behaviour in graphene multilayers, irrespective of the specific values of the tight-binding hopping parameters. It is worth noting the similarity between the effect discussed here and the phenomenology observed in a class of very different materials, namely ladders of antiferromagnetically coupled spin $1 / 2$ chains ${ }^{43}$. There, the spectrum of spin excitations also exhibits an even-odd effect, being gapped for ladders composed by an even number of chains and gapless when the number of chains is odd. Theoretical concepts that have been considered to explain the even-odd effect observed in spin ladders include topological terms related to the non-trivial Berry phase of spin $1 / 2$ and the presence of a Dirac dispersion (ubiquitous in truly one-dimensional systems), which clearly have analogies with the physics of graphene ${ }^{44}$. Nevertheless, there are important differences, for example, in spin ladders the evenodd effect is seen when going from 1D to $2 \mathrm{D}$ systems, whereas with graphene multilayers we go from $2 \mathrm{D}$ to $3 \mathrm{D}$.

An important implication of the robustness of the even-odd interaction effect, and of the experimental findings reported here, is that electron-electron interactions can be expected to determine also the properties of multilayers that are so thick to be normally considered as bulk graphite. Indeed, finding an insulating state in $4 \mathrm{LG}$ already clearly visible well above $4.2 \mathrm{~K}$, that is, more pronounced than in BLG (where it becomes visible only below $1 \mathrm{~K}$; see Supplementary Note 1), shows that the role of interactions does not become weaker, upon increasing layer thickness. This finding and the theoretical considerations made above, therefore, indicate that the commonly held notion that bulk graphite can be well understood within a single-particle model is unlikely to be correct, and that at a sufficiently lowenergy the effects of electron-electron interactions become important. These effects can easily be eclipsed in experiments on samples of microscopic size, which unavoidably contain structural defects, but when probing well-characterized, small multilayers-which can be made defect free-they become fully apparent.

\section{Methods}

Device fabrication and measurement. Samples in this study were fabricated by using polydimethylglutarimide (PMGI)-based lift-off resist (LOR, MicroChem) as a sacrificial layer ${ }^{15,45}$. Graphene flakes were mechanically exfoliated from natural graphite onto an $\sim 1-\mu$ m-thick LOR layer covering the highly doped $\mathrm{Si} / \mathrm{SiO}_{2}$ substrate (acting as a back gate), and the metallic electrodes $(10 \mathrm{~nm} \mathrm{Ti} / 70 \mathrm{~nm} \mathrm{Au})$ contacting the selected flakes were defined by using a standard microfabrication process: electron-beam lithography, deposition and lift-off. Before removing the sacrificial LOR resist locally under the flake to achieve suspension, the flake shape was defined by the exposure to an Oxygen plasma through a patterned polymethylmethacrylate resist mask (see the inset of Fig. 1a for an optical image of etching pattern). After suspension, the devices were mounted in the vacuum chamber of a Heliox ${ }^{3} \mathrm{He}$ system (Oxford Instruments; base temperature of $250 \mathrm{mK})$ and current annealed at low temperature $(T=4.2 \mathrm{~K})$ in a two-terminal 
configuration, by using shorted adjacent pair of contacts ( 1 and 2 ) and ( 3 and 4 ) as a source and drain, respectively ${ }^{15}$. As we adopt the strategy to proceed with the current annealing until all obvious manifestations of inhomogeneity in the transport properties of the device disappear, multiple annealing steps are required and the number of devices that survive the process in the end is small (in other words, we do not start with in-depth investigations of transport, which can easily take several months for a single device, as long as clear non-idealities are present in the transport characteristics of the devices).

\section{References}

1. Kotov, V. N., Uchoa, B., Pereira, V. M., Guinea, F. \& Castro Neto, A. H. Electron-electron interactions in graphene: current status and perspectives. Rev. Mod. Phys. 84, 1067-1125 (2012).

2. Elias, D. C. et al. Dirac cones reshaped by interaction effects in suspended graphene. Nat. Phys 7, 701-704 (2011).

3. Min, H., Borghi, G., Polini, M. \& MacDonald, A. H. Pseudospin magnetism in graphene. Phys. Rev. B 77, 041407 (2008).

4. Zhang, F., Min, H., Polini, M. \& MacDonald, A. H. Spontaneous inversion symmetry breaking in graphene bilayers. Phys. Rev. B 81, 041402 (2010).

5. Nandkishore, R. \& Levitov, L. Quantum anomalous Hall state in bilayer graphene. Phys. Rev. B 82, 115124 (2010).

6. Lemonik, Y., Aleiner, I. \& Fal'ko, V. I. Competing nematic, antiferromagnetic, and spin-flux orders in the ground state of bilayer graphene. Phys. Rev. B 85, 245451 (2012)

7. Jung, J., Zhang, F. \& MacDonald, A. H. Lattice theory of pseudospin ferromagnetism in bilayer graphene: competing interaction-induced quantum Hall states. Phys. Rev. B 83, 115408 (2011).

8. Velasco, Jr. J. et al. Transport spectroscopy of symmetry-broken insulating states in bilayer graphene. Nat. Nanotechnol. 7, 156-160 (2012).

9. Freitag, F., Trbovic, J., Weiss, M. \& Schönenberger, C. Spontaneously gapped ground state in suspended bilayer graphene. Phys. Rev. Lett. 108, 076602 (2012).

10. Weitz, R. T., Allen, M. T., Feldman, B. E., Martin, J. \& Yacoby, A. Broken-symmetry states in doubly gated suspended bilayer graphene. Science 330, 812-816 (2010).

11. Veligura, A. et al. Transport gap in suspended bilayer graphene at zero magnetic field. Phys. Rev. B 85, 155412 (2012).

12. Bao, W. et al. Stacking-dependent band gap and quantum transport in trilayer graphene. Nat. Phys. 7, 948-952 (2011).

13. Bolotin, K. I. et al. Ultrahigh electron mobility in suspended graphene. Solid State Commun. 146, 351-355 (2008).

14. Du, X., Skachko, I., Barker, A. \& Andrei, E. Y. Approaching ballistic transport in suspended graphene. Nat. Nanotechnol. 3, 491-495 (2008).

15. Ki, D.-K. \& Morpurgo, A. F. High-quality multiterminal suspended graphene devices. Nano Lett. 13, 5165-5170 (2013).

16. Ki, D.-K., Fal'ko, V. I., Abanin, D. A. \& Morpurgo, A. F. Observation of even denominator fractional quantum hall effect in suspended bilayer graphene. Nano Lett. 14, 2135-2139 (2014).

17. Graf, D. et al. Spatially resolved Raman spectroscopy of single- and few-layer graphene. Nano Lett. 7, 238-242 (2007).

18. Ferrari, A. C. \& Basko, D. M. Raman spectroscopy as a versatile tool for studying the properties of graphene. Nat. Nanotechnol. 8, 235-246 (2013).

19. Nguyen, T. A., Lee, J.-U., Yoon, D. \& Cheong, H. Excitation energy dependent Raman signatures of ABA- and ABC-stacked few-layer graphene. Sci. Rep. 4, 4630 (2014).

20. Berciaud, S., Potemski, M. \& Faugeras, C. Probing electronic excitations in mono- to pentalayer graphene by micro magneto-Raman spectroscopy. Nano Lett. 14, 4548-4553 (2014).

21. Guinea, F., Castro Neto, A. H. \& Peres, N. M. R. Electronic states and Landau levels in graphene stacks. Phys. Rev. B 73, 245426 (2006).

22. Latil, S. \& Henrard, L. Charge carriers in few-layer graphene films. Phys. Rev. Lett. 97, 036803 (2006).

23. Partoens, B. \& Peeters, F. M. Normal and Dirac fermions in graphene multilayers: tight-binding description of the electronic structure. Phys. Rev. B 75, 193402 (2007)

24. Koshino, M. \& Ando, T. Orbital diamagnetism in multilayer graphenes: Systematic study with the effective mass approximation. Phys. Rev. B 76, 085425 (2007)

25. Min, H. \& MacDonald, A. H. Chiral decomposition in the electronic structure of graphene multilayers. Phys. Rev. B 77, 155416 (2008).

26. Nakamura, M. \& Hirasawa, L. Electric transport and magnetic properties in multilayer graphene. Phys. Rev. B 77, 045429 (2008).

27. Koshino, M. \& McCann, E. Parity and valley degeneracy in multilayer graphene. Phys. Rev. B 81, 115315 (2010).

28. Koshino, M. \& Ando, T. Magneto-optical properties of multilayer graphene. Phys. Rev. B 77, 115313 (2008).

29. Vera-Marun, I. J. et al. Quantum Hall transport as a probe of capacitance profile at graphene edges. Appl. Phys. Lett. 102, 013106 (2013).
30. Koshino, M. \& McCann, E. Landau level spectra and the quantum Hall effect of multilayer graphene. Phys. Rev. B 83, 165443 (2011).

31. Goerbig, M. O. Electronic properties of graphene in a strong magnetic field. Rev. Mod. Phys. 83, 1193-1243 (2011).

32. Koshino, M. Electronic transmission through $A B-B A$ domain boundary in bilayer graphene. Phys. Rev. B 88, 115409 (2013).

33. San-Jose, P., Gorbachev, R. V., Geim, A. K., Novoselov, K. S. \& Guinea, F. Stacking Boundaries and Transport in Bilayer Graphene. Nano Lett. 14, 2052-2057 (2014).

34. Mayorov, A. S. et al. Interaction-driven spectrum reconstruction in bilayer graphene. Science 333, 860-863 (2011).

35. Apalkov, V. M. \& Chakraborty, T. Controllable driven phase transitions in fractional quantum Hall states in bilayer graphene. Phys. Rev. Lett. 105, 036801 (2010).

36. Papić, Z., Abanin, D. A., Barlas, Y. \& Bhatt, R. N. Tunable interactions and phase transitions in Dirac materials in a magnetic field. Phys. Rev. B 84, 241306 (2011).

37. Papić, Z. \& Abanin, D. A. Topological Phases in the zeroth Landau level of bilayer graphene. Phys. Rev. Lett. 112, 046602 (2014).

38. Kou, A. et al. Electron-hole asymmetric integer and fractional quantum Hall effect in bilayer graphene. Science 345, 55-57 (2014).

39. Maher, P. et al. Tunable fractional quantum Hall phases in bilayer graphene. Science 345, 61-64 (2014).

40. Kharitonov, M. Canted antiferromagnetic phase of the $v=0$ quantum Hall state in bilayer graphene. Phys. Rev. Lett. 109, 046803 (2012).

41. Maher, P. et al. Evidence for a spin phase transition at charge neutrality in bilayer graphene. Nat. Phys. 9, 154-158 (2013).

42. Young, A. F. et al. Tunable symmetry breaking and helical edge transport in a graphene quantum spin Hall state. Nature 505, 528-532 (2014).

43. Dagotto, E. \& Rice, T. M. Surprises on the way from one- to two-dimensional quantum magnets: the ladder materials. Science 271, 618-623 (1996).

44. Castro Neto, A. H., Guinea, F., Peres, N. M. R., Novoselov, K. S. \& Geim, A. K. The electronic properties of graphene. Rev. Mod. Phys. 81, 109-162 (2009).

45. Tombros, N. et al. Large yield production of high mobility freely suspended graphene electronic devices on a polydimethylglutarimide based organic polymer. J. Appl. Phys. 109, 093702 (2011).

\section{Acknowledgements}

A.L.G., D.-K.K. and A.F.M. thank Nicolas Ubrig for help with measuring Raman spectra of the devices at zero magnetic field, and A. Ferreira for technical support. A.F.M. gratefully acknowledges the support from the SNF and the NCCR QSIT. M.K. is funded by JSPS Grant-in-Aid for Scientific Research Nos. 24740193 and 25107005 . The work has been also supported by the EU under the Graphene Flagship (A.F.M., C.F. and E.M.) and by the European Research Council (A.A.L.N. and M.P.).

\section{Author contributions}

A.L.G. and D.-K.K. fabricated the devices, performed the electrical transport measurements and analysed the data. C.F., A.N., A.L.G. and M.P. carried out the magneto-Raman measurements and analysed the results. M.K. and E.M. contributed to the theoretical analysis, and M.K. performed theoretical calculations of the effect of staggered interlayer potential in multilayer graphene. A.F.M. supervised the work, discussed the transport experiments with A.L.G. and D.-K.K. and interpreted the results in collaborations with A.L.G., D.-K.K., M.K. and E.M. The paper and the supplementary information were written by A.F.M., A.L.G., D.-K.K. and M.K, who included suggestions and comments from all the authors.

\section{Additional information}

Supplementary Information accompanies this paper at http://www.nature.com/ naturecommunication

Competing financial interests: The authors declare no competing financial interests.

Reprints and permission information is available at http://npg.nature.com/ reprintsandpermissions.

How to cite this article: Grushina, A.L. et al. Insulating state in tetralayers reveals an even-odd interaction effect in multilayer graphene. Nat. Commun. 6:6419 doi: 10.1038/ncomms7419 (2015).

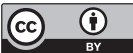

This work is licensed under a Creative Commons Attribution 4.0 International License. The images or other third party material in this article are included in the article's Creative Commons license, unless indicated otherwise in the credit line; if the material is not included under the Creative Commons license, users will need to obtain permission from the license holder to reproduce the material. To view a copy of this license, visit http://creativecommons.org/licenses/by/4.0/ 